\title{
Effect of Chair aerobics as low intensity exercise training on heart rate, blood pressure and six minute walk distance in post coronary artery bypass graft surgery patients through phase I cardiac rehabilitation
}

\author{
Shaili Thapa, ${ }^{1}$ Renu B.Pattanshetty ${ }^{2}$ \\ ${ }^{1}$ Shahid Gangalal National Heart Centre, Bansbari, Kathmandu, Nepal \\ ${ }^{2}$ KLEU Institute of Physiotherapy, Belgaum, Karnataka, India \\ Corresponding Author: Shaili Thapa, Shahid Gangalal National Heart Centre, Bansbari,Kathmandu, Nepal, Email: shaili_4@hotmail.com
}

\begin{abstract}
Background and Aims: Cardiac Rehabilitation after Coronary arteries bypass graft (CABG) surgery is known to have several favorable effects. Aerobics exercise in the form of chair aerobics shown to have beneficial effects with little evidence. The purpose of the present study was to demonstrate the effect of chair aerobics as low intensity exercise training in heart rate, blood pressure and six minute walk distance in post CABG patients during phase I cardiac rehabilitation.

Methods: It was a single centre, prospective study carried out in Indian tertiary care set-up. Fifty patients post CABG patients were included. Chair aerobics as a low intensity exercise training was given. Pre and post-exercise outcome measurements in the form of heart rate, blood pressure and six-minute walk distance test were recorded.

Results: The study showed statistically significant difference in systolic blood pressure $(\mathrm{p}=0.018)$, heart rate $(\mathrm{p} \leq 0.001)$ and increase in six minute walk distance $(\mathrm{p} \leq 0.001)$.

Conclusion: : Chair aerobics as low intensity exercise training demonstrated improvement in heart rate, systolic blood pressure and six minute walk distance test in subjects with CABG through phase I cardiac rehabilitation.
\end{abstract}

Key words: CABG, Cardiac rehabilitation Phase I, Chair aerobics

\begin{abstract}
Introduction
Coronary artery bypass graft (CABG) is a preferred treatment option for multi-vessel disease and Left main disease patients. It was first performed in India in $1975 .{ }^{1}$ Cardiac Rehabilitation (CR) is an interdisciplinary team approach to patients with functional limitations secondary to heart disease ${ }^{2}$ and known to have favorable effects in post CABG patients. Physical training enhances exercise tolerance, activity of daily living, and quality of life. ${ }^{3}$ According to the American College of Sports Medicine (ACSM), any sport or activity that works large groups of muscles, is continually maintained and performed rhythmically, is defined as an aerobic or cardiovascular exercise. ${ }^{4}$ Chair Aerobics are the aerobic exercises performed while sitting in a chair or wheelchair. ${ }^{5}$ There is hardly any evidence to demonstrate effect of chair aerobics on heart rate, blood pressure and six-minute walk distance test (SMWD) in post CABG patients during phase I Cardiac Rehabilitation. Our study aim to evaluate the effect of chair aerobics, as low intensity exercise training on blood pressure, heart rate and SMWD.
\end{abstract}

\section{Methods}

The study was a pre post experimental design with random selection of CABG subjects using a non probability sampling method. The data was collected from the post operative cardiac general ward in Kels Hospital, Belgaum,Karnataka, India during the study period extending for twelve months. Ethical approval for the study was granted by the Institutional Ethical Committee and the procedures were conducted according to the declaration of Helsinki. All post operative CABG subjects age group of 35-65yrs, mentally stable, subjects willing to participate in the study enrolled in phase I cardiac rehabilitation were included in the study. Subjects were excluded if they fell in the high risk category according to American College of Sports Medicine risk stratification guidelines, ${ }^{6}$ conditions that were contraindicated to exercise training (American College of Sports Medicine guidelines), ${ }^{7}$ with drains, $\mathrm{CABG}$ with valve replacement, $\mathrm{CABG}$ with pacemaker and subjects undergoing psychiatric treatment. Sample size was calculated with a error of 80 and level of significance of $\mathrm{p} \leq 0.05$ and assuming fall out rate of $10 \%$ of subjects. Statistical analysis of the data was done using "SPSS" windows version 20.0. Demographic data of the subjects i.e. age, gender, weight, height, body mass index, occupation, risk factors and diet history were analyzed using statistical measures like mean, standard deviation and percentage. Student paired ' $t$ " test was used to analyze the outcome measures (pre and post) of the subjects in the study. Probability value $(\mathrm{p}) \leq 0.05$ was considered statistically significant. 


\section{Procedure:}

All the subjects were initially examined for compliance with inclusion and exclusion criteria. Demographic and baseline data of outcome variables of each subject was recorded. Subjects were given incentive spirometry in the form of breathing exercises. Warm up exercises in the form of active range of motion exercises for upper limb and lower limb was given with 5 repetitions of each large joints. This was followed by short session of chair aerobics exercises as per the intensity tolerated by the subject using RPE(Rate of perceive exertion) Borg's scale. ${ }^{8}$ The exercise session ended with stretches and range of motion exercises for upper limb and lower limb as cool down exercises. The entire session did not increase its pre planned time limits. Immediately after the exercises the Heart rate, Blood Pressure and Rate Pressure Products were measured. After the 3 min recovery period post exercises, a six minutes walk test was performed as per ATS guidelines 9 to check the endurance calculated in the form of distance covered by the subject at the day of discharge.

\section{Exercise protocol}

\section{$0-4$ days:}

Incentive spirometry in the form of breathing exercises for 5 times.

Active range of motion exercises for shoulder joint, elbow joint, wrist joint, hip joint, knee joint and ankle joint for 5 times each.

Chair aerobic exercises were given as below:

1. Alternate heel digs with bilateral biceps curl -8 repetitions

2. $\mathrm{V}$ - step with bilateral hammer curls -8 repetitions

3. Lateral step touch with bilateral biceps curl -8 repetitions

4. Knee lift -8 repetitions

The intensity of the exercises was low and as tolerated by the subject on the basis of RPE Borg's scale. 8 The total time duration was 15 minutes.

\section{5th - 8th days:}

Incentive spirometry in the form of breathing exercises for 8 times.

Active ROM exercises for shoulder joint, elbow joint, wrist joint, hip joint, knee joint and ankle joint for 8 times each. Chair aerobic exercises were given as below:

1. Alternate heel digs with bilateral arm curl -16 repetitions

2. V-Step with bilateral hammer curls -16 repetitions

3. Lateral step touch with bilateral arm curl -16 repetitions

\section{Knee lift -16 repetitions}

The intensity of the exercises was low and as tolerated by the subject on the basis of RPE Borg's scale. 8 The total time duration was 20 minutes.

\section{9th days - till discharged from hospital:}

Incentive spirometry in the form of breathing exercises for 10 times.

Active ROM exercises for shoulder joint, elbow joint, wrist joint, hip joint, knee joint and ankle joint for 10 times each. Chair aerobic exercises were given as below:

1. Alternate heel digs with bilateral arm curl -32 repetitions

2. V-step with bilateral hammer curls - 32 repetitions

3. Lateral step touch with bilateral arm curl -32 repetitions

4. Knee lift -32 repetitions

The intensity of the exercises was low and as tolerated by the subject on the basis of RPE Borg's scale. ${ }^{8}$ The total time duration was 30 minutes.

\section{Measurement of outcome variables}

Hemodynamic variables were measured as per the ACSM guidelines. ${ }^{10}$

- Blood pressure: It was measured as per the ACSM guidelines.

- Heart Rate: was done by palpation method.

- Six minute walk test was carried out as per ATS guidelines. ${ }^{9}$

\section{Results}

A total of eighty two (82) subjects with post CABG surgery, were screened in Phase I Cardiac rehabilitation program during the study recruitment period, out of which sixty (60) were enrolled into the study based on the eligibility criteria. Fifty (50) subjects completed exercise training protocol. The results of the study were based on the data analyzed for fifty (50) subjects. Demographic data of the subjects are outlined in Table 1 . Mean age of subjects was $56.17 \pm 7.17$. Fourty $(80 \%)$ subjects were males and $10(20 \%)$ were females. Outcome measures pre and post intervention are demonstrated in Table 2. There was significant difference in the systolic blood pressure $(\mathrm{p}=0.018)$ where as diastolic blood pressure did not demonstrate any changes in between pre and post intervention. The mean difference of heart rate between pre and post intervention was statistically significant $(\mathrm{P} \leq 0.001)$. Distance covered in six minute walk test improved significantly $(\mathrm{p} \leq 0.001)$ after the intervention with a mean difference of 91.86 meters.

\section{Figure 1: Consort Flow Study Chart}

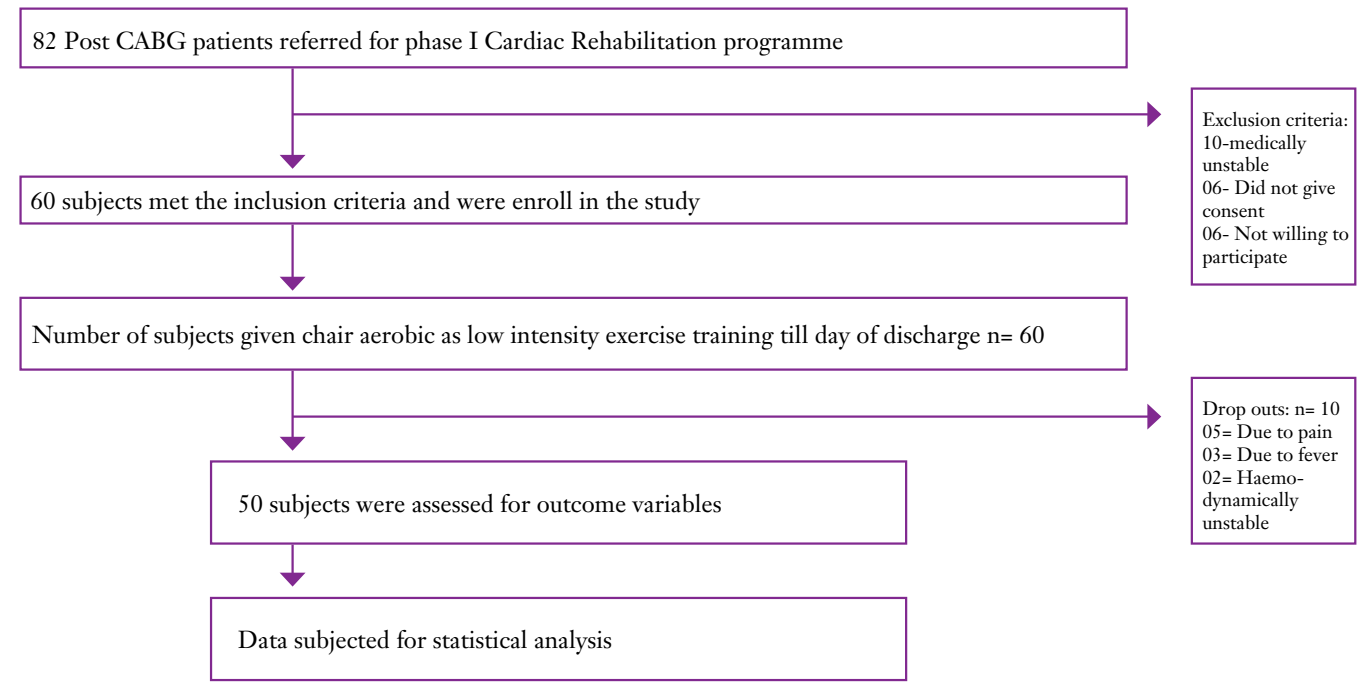


Table 1: Demographic distribution of subjects participating in the study

\begin{tabular}{|c|c|c|c|c|c|}
\hline S.No. & Demographic Characteristics & & Number & Percentage (\%) & Mean \pm SD \\
\hline \multirow{3}{*}{1.} & \multirow{3}{*}{ Age groups (Years) } & $40-50$ & 11 & 22 & \multirow{3}{*}{$56.17 \pm 7.17$} \\
\hline & & $51-60$ & 20 & 40 & \\
\hline & & $61-65$ & 19 & 38 & \\
\hline \multirow[t]{2}{*}{2.} & Gender & Male & 40 & 80 & \multirow{5}{*}{$62.62 \pm 10.37$} \\
\hline & & Female & 10 & 20 & \\
\hline \multirow[t]{3}{*}{3.} & Weight (Kg) & $\leq 55$ & 17 & 34 & \\
\hline & & $56-70$ & 20 & 40 & \\
\hline & & $>70$ & 13 & 26 & \\
\hline \multirow[t]{3}{*}{4.} & Height (cm) & $\leq 150$ & 10 & 20 & \multirow{3}{*}{$158.36 \pm 7.68$} \\
\hline & & $151-160$ & 24 & 48 & \\
\hline & & $161-170$ & 16 & 32 & \\
\hline \multirow{5}{*}{5.} & \multirow{5}{*}{ BMI (Kg/M2) } & $<18.5$ & 0 & 0.0 & \multirow{5}{*}{$24.94 \pm 3.34$} \\
\hline & & $18.5-24.9$ & 24 & 48 & \\
\hline & & $25-29.9$ & 23 & 46 & \\
\hline & & $30-34.9$ & 3 & 6 & \\
\hline & & $\geq 35$ & 0 & 0.0 & \\
\hline \multirow{6}{*}{6.} & \multirow{6}{*}{ Occupation } & Labourer & 8 & 16 & \\
\hline & & Farmer & 13 & 26 & \\
\hline & & Service & 12 & 24 & \\
\hline & & Businessman & 7 & 14 & \\
\hline & & Housewife & 8 & 16 & \\
\hline & & Teacher & 2 & 4 & \\
\hline \multirow{6}{*}{7.} & \multirow{6}{*}{ Risk factors } & Family History & 10 & 20 & \\
\hline & & Cigarette Smoking & 8 & 26 & \\
\hline & & Hypertension & 31 & 62 & \\
\hline & & Dyslipidemia & 26 & 52 & \\
\hline & & Diabetic Mellitus & 25 & 50 & \\
\hline & & Obesity & 3 & 6 & \\
\hline
\end{tabular}

Table 2: Outcome measures of the subjects participating in the study

\begin{tabular}{|c|c|c|c|c|c|}
\hline Outcome measures & $\begin{array}{l}\text { Baseline } \\
\text { Mean } \pm \text { SD }\end{array}$ & $\begin{array}{l}\text { Post-test } \\
\text { Mean } \pm \text { SD }\end{array}$ & $\begin{array}{l}\text { Mean } \\
\text { difference }\end{array}$ & $\mathrm{t}$-value & 'p' value \\
\hline $\begin{array}{l}\text { Systolic Blood Pressure } \\
(\mathrm{mmHg})\end{array}$ & $120.52 \pm 9.71$ & $116.64 \pm 9.73$ & -3.88 & 2.45 & 0.018 \\
\hline $\begin{array}{l}\text { Diastolic Blood Pressure } \\
(\mathrm{mmHg})\end{array}$ & $77.32 \pm 7.67$ & $75.04 \pm 7.23$ & -2.28 & 1.83 & 0.072 \\
\hline Heart rate (beats/min) & $90.01 \pm 6.24$ & $84.74 \pm 6.1$ & -5.27 & 5.55 & $<0.001$ \\
\hline Six minute walk distance (mts) & $97.22 \pm 76.51$ & $189.08 \pm 122.15$ & 91.86 & -10.27 & $<0.001$ \\
\hline
\end{tabular}

Level of significance $\mathrm{p} \leq 0.05$ 


\section{DISCUSSION}

The present study was conducted to study the effect of chair aerobics as low intensity exercise training on BP, HR and SMWD in Post CABG patients during phase I Cardiac Rehabilitation. There was significant difference in the systolic blood pressure in the present study where as diastolic blood pressure did not demonstrate any changes in between pre and post intervention. Fagard RH, demonstrated no changes in diastolic blood pressure with slight change in systolic blood pressure after dynamic physical training from three to five times per week during 30-60 min per session. 11 Another study by Baines CL et al. demonstrated significant changes in both systolic and diastolic blood pressure after twelve weeks low intensity exercise conditioning program..$^{12}$

Heart rate is primarily mediated by the direct activity of the autonomic nervous system with predominance of vagal activity (parasympathetic) at rest that is progressively inhibited from the onset of exercise. Aerobic training has shown to lower resting heart rate due to higher parasympathetic activity. ${ }^{13}$ Present study demonstrated post intervention decrease in heart rate which is similar to the study conducted by Forjaz C.M.L et al. ${ }^{14}$ However, possible explanation for the decrease in heart rate may be due to low intensity exercises which may induce a reduction in sympathetic nerve activity to the heart and blood vessels during the recovery period.

The six minute walk test is a simple and feasible test.9 The six minute walk test in a cardiac rehabilitation population is reliable and valid measures to check the functional exercise ability. ${ }^{15} \mathrm{~A}$ study by Nilsson $\mathrm{BB}$ et al. in congestive heart failure subjects demonstrated significant improvements in walking distance after thirty two sessions of high intensity aerobic training. ${ }^{16}$ Larsen AI et al. checked sub maximal exercise capacity in subjects with heart failure by using six minute walk distance and found significant improvement in sub maximal exercise capacity following twelve weeks of exercise training. ${ }^{17}$ The twelve week progressive quadriceps resistance training in patients with stable coronary heart failure has shown improvements in the muscle strength as well as the functional capacity by using six minute walk distance. ${ }^{18}$ In the present study, distance covered by the subjects in six minute walk test, significantly improved after the intervention similar to the study conducted by Hirschhorn $\mathrm{AD}$ et al where Phase 1 walking exercise program performed under direct physiotherapy supervision significantly improved sub-maximal exercise capacity at discharge from hospital using six-minute walking assessment distance. 19 Hence, the clinical importance of this early fitness lies in the potential for earlier improvements in functional activity, and expedited referral to exercise-based outpatient (Phase II) cardiac rehabilitation (CR) programs.

Though the present study consisted of small sample size with heterogeneous population, it demonstrated that chair aerobics as low intensity exercises may be helpful in improving HR, SBP and SMWD in subjects after CABG surgery with low and moderate risk. Future studies should include larger sample size, with longer follow up period to assess the long term benefits in other phases, homogenous group to find out gender differences in the outcome measures and multi-centric trials of a similar set up.

\section{CONCLUSION}

Chair aerobics as low intensity exercises may be used in low to moderate risk subjects of CABG enrolled in phase I rehabilitation in improving their functional capacity.

\section{REFERENCES:}

1. Kaul U, Bhatia V.Perspective on coronary interventions \& cardiac surgeries in India. Indian J Med Res 2010 ;132:543548.

2. Braddom RL. Physical medicine and rehabilitation. 3rd ed. Missouri, USA: Elsevier Limited; 2007.709-738.

3. Hitoshi A, Tetsuya T, Hiroshi H, Makoto K. Short-Term physical training improves ventilatory response to exercise after coronary arterial bypass surgery. Jpn Circ J 2001; 65:419-423.

4. The ACSM definition of cardiovascular exercise. Available from: URL: http://healthyliving.azcentral.com/acsmdefinition-cardiovascular-exercise-18723.html.

5. ChairAerobics.Availablefrom:URL:http://www. medicaldictionary.thefreedictionary.com/Chair +Aerobics.

6. More B, Wilkins WL. "ACSM Coronary Artery Disease Risk Factor Thresholds". In Textbook of American College of Sports Medicine: Guidelines for Exercise Testing and Prescription. 8th ed. Heritage Publications; 2010. p. 24.

7. Mahler DA, Froelicher VF, Miller NH, York TD. ACSM's Guidelines for Exercise Testing and Prescription. 5th ed. Williams and Wilkins; 1995. p. 42.

8. Borg, GV: Psychophysical basis of perceived exertion. Med Sci Sports Exer 1982;14: 377-381.

9. American Thoracic Society. ATS Statement: Guidelines for the Six- Minute Walk test. AMJ Respir Crit Care Med 2002 ; 166:111-117.

10. More B, Wilkins WL. In resting and exercise blood pressure and heart rate: ACSM'S Health -Related Physical Fitness Assessment Manual. 2nd ed. Lippincott Williams and Wilkins: Chapter 3; 2008. p. 27.

11. Fagard RH. Exercise characteristics and the blood pressure response to dynamic physical training. Med. Sci. Sports Exerc. 2001;33 (6):S484-S492.

12. Baines CL, Brown C.A. Effects of low-intensity exercise conditioning on blood pressure, heart rate, rate-pressureproduct and cardiac autonomic function inhypertensive women. European Journal of Cardiovascular Nursing, 2009;8:S1-S52.

13. Almeida MB, Araujo CGS. Effects of aerobic training on heart rate. Rev. Bras. Med. Esporte 2003; 9:113-118.

14. Forjaz CLM, Matsudaira Y, Rodrigues FB, et al.Post-exercise changes in blood pressure, heart rate and rate pressure product at different exercise intensitiesin normotensive humans. Brazilian journal of medical and biologcal research 1998:31(10); 1247-1255.

15. Hamilton DM, Haennel RG. Validity and reliability of the 6-minute walk test in a cardiac rehabilitation population. Journal of Cardiopulmonary Rehabilitation 2000; 20(3):156164.

16. Nilsson BB, WestheimA, Risberg MA. Long term effect of a group based high intensity aerobic interval training program in patients with chronic heart failure. Am J Cardiol, 2008; 102(9):1220-1224.

17. Larsen AI, Aarsland T, Kristiansen M, et. al.Assessing the effect of exercise training in men with heart failure, comparison of maximal, submaximal and endurance exercise protocol. Eur Heart J 2001; 22(8):684-692.

18. JankowskaEA, Wegrzynowska K, Superlak M, et. al. The twelve week progressive quadriceps resistance training improves muscle strength, exercise capacity and quality of life in patients with stable coronary heart failure. Int J Cardiol 2008;130(1):36-43.

19. Hirschhorn AD, Richards D, Mungovan SF,et. Al. 
Supervised moderate intensity exercise improves distance walked at hospital discharge following coronary artery bypass graft surgery-A randomised controlled trial. Heart, Lung and Circulation 2008; 17:129-138.

Cite this article as: Shaili Thapa, Renu B.Pattanshetty. Effect of Chair aerobics as low intensity exercise training on heart rate, blood pressure and six minute walk distance in post coronary artery bypass graft surgery patients through phase I cardiac rehabilitation. Nepalese Heart Journal 2016;13(1):-19-24. 